\title{
Trees and mapping class groups
}

\author{
Richard P. Kent IV*, Christopher J. Leininger†, Saul Schleimer ${ }^{\ddagger}$
}

October 30, 2008

\begin{abstract}
There is a forgetful map from the mapping class group of a punctured surface to that of the surface with one fewer puncture. We prove that finitely generated purely pseudo-Anosov subgroups of the kernel of this map are convex cocompact in the sense of B. Farb and L. Mosher. In particular, we obtain an affirmative answer to their question of local convex cocompactness of K. Whittlesey's group.

In the course of the proof, we obtain a new proof of a theorem of I. Kra. We also relate the action of this kernel on the curve complex to a family of actions on trees. This quickly yields a new proof of a theorem of J. Harer.
\end{abstract}

\section{Introduction}

Let $S=S_{g, m}$ be an orientable surface of genus $g$ with $m$ punctures and assume throughout that the complexity $\xi(S)=3 g-3+m$ is at least 1 . Let $(S, z)$ denote the surface $S$ equipped with a marked point $z$. There is a homomorphism from the mapping class group of $(S, z)$ to that of $S$ which fits into J. Birman's exact sequence $[3,4]$

$$
1 \rightarrow \pi_{1}(S, z) \rightarrow \operatorname{Mod}(S, z) \rightarrow \operatorname{Mod}(S) \rightarrow 1,
$$

see $\S 2$. We use this sequence to view $\pi_{1}(S, z)$ as a subgroup of $\operatorname{Mod}(S, z)$.

Our main theorem answers Question 6 of [13].

Theorem 6.1. If $G<\pi_{1}(S, z)<\operatorname{Mod}(S, z)$ is finitely generated and purely pseudo-Anosov, then $G$ is convex cocompact.

${ }^{*}$ This research was partially conducted during the period the first author was employed by the Clay Mathematics Institute as a Liftoff Fellow. This author was also supported by a Donald D. Harrington Dissertation Fellowship and an NSF postdoctoral fellowship.

${ }^{\dagger}$ partially supported by NSF grant DMS-0603881

${ }^{\ddagger}$ partially supported by NSF grant DMS-0508971

${ }^{0}$ This work is in the public domain. 
Convex cocompactness for subgroups of the mapping class group was defined by B. Farb and L. Mosher in [8] via the action on Teichmüller space by way of analogy with Kleinian groups and their action on hyperbolic space: a subgroup is convex cocompact if it has a quasiconvex orbit in the Teichmüller space. Their work exhibits an intimate connection between convex cocompactness of a subgroup of the mapping class group and the geometry of the associated surface group extension. This concept was studied further by the first two authors in [13], extending the analogy with Kleinian groups; and by U. Hamenstädt in [9], where the connection to surface group extensions was strengthened. We note that Theorem 6.1 provides the first nontrivial examples of convex cocompact groups that do not arise from a combination or ping-pong argument.

In terms of the analogy with Kleinian groups, Theorem 6.1 should be compared with a theorem of G. P. Scott and G. A. Swarup [18]: finitely generated subgroups of infinite index in fiber subgroups of fibered hyperbolic 3-manifold groups are geometrically finite. Indeed, the exact sequence of such a fibration

$$
1 \rightarrow \pi_{1}(S, z) \rightarrow \pi_{1}(M, z) \rightarrow \mathbb{Z} \rightarrow 1
$$

injects into Birman's sequence, and the subgroups covered by Theorem 6.1 are natural analogues of those considered by Scott and Swarup.

Convex cocompact groups are necessarily finitely generated and virtually purely pseudo-Anosov [8]. An important question is whether the converse holds - this is Question 1.5 of [8] (see also Problem 3.4 of [17]), asked by Farb and Mosher for free groups. A negative answer would imply a negative answer to M. Gromov's Question, Question 1.1 of [2], regarding necessary and sufficient conditions for a group to be word hyperbolic. See Section 8 of [14] for a discussion of the connection between Gromov's question and convex cocompactness.

Question 6 of [13] is a natural test question for Question 1.5 of [8] as the necessary and sufficient condition for an element in $\pi_{1}(S, z)$ to be pseudo-Anosov as an element of $\operatorname{Mod}(S, z)$ is a topological one, and not a priori related to any algebraic structure. I. Kra discovered this necessary and sufficient condition [15] - see Theorem 4.2 here - and his proof of sufficiency is Teichmüller theoretic (necessity is obvious). We give an alternative proof here based entirely on topological and group theoretic considerations, see $§ 4$.

The class of groups covered by Theorem 6.1 also includes the test case proposed by Farb and Mosher in Question 1.6 of [8] (see also Problem 3.5 of [17]). These are the finitely generated subgroups of K. Whittlesey's groups. Recall that Whittlesey's groups are normal purely pseudo-Anosov subgroups of the mapping class groups of the sphere with $n \geq 5$ punctures and of the closed genus -2 surface.

Corollary. Whittlesey's groups are locally convex cocompact: finitely generated subgroups are convex cocompact.

Proof of the Corollary from Theorem 6.1. It suffices to prove the theorem for Whittlesey's subgroups of $\operatorname{Mod}\left(S_{0, n}\right)$, as there is a surjection

$$
\operatorname{Mod}\left(S_{2,0}\right) \rightarrow \operatorname{Mod}\left(S_{0,6}\right)
$$


with order-two central kernel [5], and an isometry of Teichmüller spaces which is equivariant with respect to this "virtual isomorphism."

We can view any one of the punctures of $S_{0, n}$ as being obtained from $S_{0, n-1}$ by removing a marked point $z$. There are thus $n$ different Birman sequences and so $n$ surjective homomorphisms $\operatorname{Mod}\left(S_{0, n}\right) \rightarrow \operatorname{Mod}\left(S_{0, n-1}\right)$. The intersection of these kernels is Whittlesey's group, and hence lies in $\pi_{1}\left(S_{0, n-1}\right)<\operatorname{Mod}\left(S_{0, n}\right)$. Any finitely generated subgroup of Whittlesey's group is thus also a finitely generated purely pseudo-Anosov subgroup of $\pi_{1}\left(S_{0, n-1}\right)$. Since $n \geq 5$, Theorem 6.1 implies that such a subgroup is convex cocompact.

The proof of Theorem 6.1 relies on a characterization of convex cocompactness discovered by the first two authors [13] and, independently, by Hamenstädt $[9]$ in terms of the action on the curve complex $\mathcal{C}$. We are thus lead to a study of the action of $\pi_{1}(S, z)<\operatorname{Mod}(S, z)$ on the curve complex of $(S, z)$ (equivalently, that of $S \backslash\{z\}$ ), and there is an interesting observation regarding this action that we now describe.

If $S$ is closed $(m=0)$, then there is a map of curve complexes $\Pi: \mathcal{C}(S, z) \rightarrow$ $\mathcal{C}(S)$; see $\S 2.2$. In general, the desired map is not globally well-defined as essential simple closed curves on $(S, z)$ may become peripheral in $S$. In this case we restrict $\Pi$ to the largest subcomplex on which it is well-defined, denoted $\widehat{\mathcal{C}}(S, z)$. The fibers of this map are invariant under the action of $\pi_{1}(S, z)<\operatorname{Mod}(S, z)$, and have a simple geometric description:

Theorem 7.1. The fiber of $\Pi$ over a point in the interior of a simplex $v \subset \mathcal{C}(S)$ is $\pi_{1}(S, z)$-equivariantly homeomorphic to the tree $T_{v}$ determined by $v$.

The tree $T_{v}$ is the tree dual to the multi-curve $v$ equipped with its action by $\pi_{1}(S, z)$; see $\S 7$. This is the Bass-Serre tree for the splitting of $\pi_{1}(S, z)$ determined by the multi-curve $v$.

A consequence of the theorem is the following fact due to J. Harer [10] - a new proof of this is due to A. Hatcher and K. Vogtmann [11].

Corollary 1.1 (Harer). With the polyhedral topologies, $\mathcal{C}(S)$ and $\widehat{\mathcal{C}}(S, z)$ are homotopy equivalent.

The corollary is proven using a section of $\Pi$ described by Harer and performing the straight-line homotopy to the section along the fibers given by Theorem 7.1. See $\S 7$.

Acknowledgments. The authors thank Yair Minsky, Ursula Hamenstädt, Alan Reid, and Ben Wieland for helpful and interesting conversations. The second author thanks the Max-Plank-Institut für Mathematik in Bonn for its hospitality during part of this work. We also thank Andy Putman for his careful reading and comments on an earlier version of this paper and the referee for providing helpful organizational advice. 


\section{Definitions and conventions}

We have chosen to work with the surface $(S, z)$ marked with $z$ rather than $S \backslash\{z\}$ as this is generally more convenient. However, occasionally our arguments are clarified by working with $S \backslash\{z\}$. When this is the case, we refer to the puncture obtained by removing $z$ as the $z$-puncture.

We say that a closed curve in $S$ is nontrivial if it is homotopically nontrivial in $S$ and essential if it is nontrivial and nonperipheral, that is, not homotopic into every neighborhood of a puncture. These definitions are extended to $(S, z)$ by defining a closed curve in $(S, z)$ to be a closed curve in $S$ which is contained in $S \backslash\{z\}$. A closed curve in $(S, z)$ is then nontrivial (respectively, essential) if it is so in $S \backslash\{z\}$. Isotopy in $(S, z)$ means isotopy in $S$ fixing $z$. Thus nontrivial and essential simple closed curves in $(S, z)$ are isotopic if and only if they are isotopic in $S \backslash\{z\}$.

We fix a complete finite area hyperbolic metric on $S$ and let $p: \widetilde{S} \rightarrow S$ denote the universal covering. The hyperbolic metric on $S$ pulls back to one on $\widetilde{S}$ making $\widetilde{S}$ isometric to the hyperbolic plane.

We view $\pi_{1}(S)$ as the group of covering transformations of the universal covering $p: \widetilde{S} \rightarrow S$ and fix this action once and for all. A point $\widetilde{z} \in p^{-1}(z)$ determines an isomorphism of $\pi_{1}(S)$ with the fundamental group $\pi_{1}(S, z)$. We fix a basepoint $\widetilde{z} \in p^{-1}(z)$, and hence an isomorphism $\pi_{1}(S) \cong \pi_{1}(S, z)$.

\subsection{Mapping class groups and Birman's sequence}

The mapping class group of $S$ is the $\operatorname{group} \operatorname{Mod}(S)=\pi_{0}\left(\operatorname{Diff}^{+}(S)\right)$, where $\operatorname{Diff}^{+}(S)$ is the group of orientation preserving diffeomorphisms of $S$ that fix each of the punctures. We define $\operatorname{Mod}(S, z)$ to be $\pi_{0}\left(\operatorname{Diff}^{+}(S, z)\right)$, where $\operatorname{Diff}^{+}(S, z)$ is the group of orientation preserving diffeomorphisms of $S$ that fix each puncture and that also fix $z$. There is a canonical isomorphism $\operatorname{Mod}(S, z) \cong \operatorname{Mod}(S \backslash\{z\})$.

Birman's exact sequence $[3,4]$ relates the mapping class group of $S$ with that of $(S, z)$ and $\pi_{1}(S, z)$. Namely

$$
1 \rightarrow \pi_{1}(S, z) \rightarrow \operatorname{Mod}(S, z) \rightarrow \operatorname{Mod}(S) \rightarrow 1 .
$$

To describe the inclusion $\pi_{1}(S, z) \rightarrow \operatorname{Mod}(S, z)$ concretely, we first represent an element of $\pi_{1}(S, z)$ by a loop $\gamma$ based at $z$. Writing $\gamma:[0,1] \rightarrow S$ with $\gamma(0)=\gamma(1)=z$, let $h_{t}: S \rightarrow S, t \in[0,1]$, be any isotopy such that $h_{0}=\operatorname{Id}_{S}$ and $\gamma(1-t)=h_{t}(z)$ for all $t \in[0,1]$. Since $h_{1}(z)=z$, the map $h_{1}$ determines a mapping class in $\operatorname{Mod}(S, z)$, and this is the image of $\gamma$ in $\operatorname{Mod}(S, z)$ in the exact sequence. It is clear that the isotopy $h_{t}$ may be constructed so that the diffeomorphism $h_{1}$ is supported on any given neighborhood of the curve $\gamma \subset S$.

For clarity, we write $h_{\gamma}$ for the diffeomorphism or mapping class associated to $\gamma \in \pi_{1}(S, z)$.

Any element $f \in \operatorname{Mod}(S)$ determines an outer automorphism $f_{*}$ of $\pi_{1}(S, z)$, and this defines a homomorphism $\operatorname{Mod}(S) \rightarrow \operatorname{Out}\left(\pi_{1}(S, z)\right)$ - the codomain is $\operatorname{Out}\left(\pi_{1}(S, z)\right)$ rather than $\operatorname{Aut}\left(\pi_{1}(S, z)\right)$ as representatives of $f$ need not fix the 
basepoint. The Dehn-Nielsen Theorem states that this is an isomorphism onto an index-two subgroup when $S$ is closed (see Stillwell's appendix to [7]), but in general it is only injective [20]. Working with $\operatorname{Mod}(S, z)$ erases the difficulty of moving basepoints and there is an injection $\operatorname{Mod}(S, z) \rightarrow \operatorname{Aut}\left(\pi_{1}(S, z)\right)$. Since $\pi_{1}(S, z)$ has trivial center, $\pi_{1}(S, z) \cong \operatorname{Inn}\left(\pi_{1}(S, z)\right)$ and Birman's exact sequence injects into the classical short exact sequence associated to $\operatorname{Aut}\left(\pi_{1}(S, z)\right)$ :

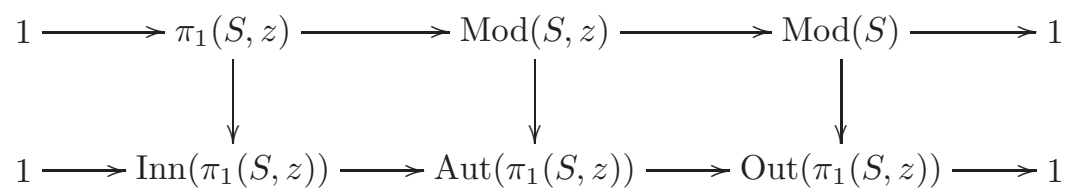

All of the squares commute, and, for any $\alpha, \gamma \in \pi_{1}(S, z)$, the first one becomes

$$
\left(h_{\gamma}\right)_{*}(\alpha)=\gamma \alpha \gamma^{-1} .
$$

\subsection{Curve complexes}

The simplicial curve complex of $S$ will be denoted $\mathcal{C}^{\Delta}(S)$. This is an abstract simplicial complex defined on the set of isotopy classes of essential simple closed curves on $S$ by declaring a set $v=\left\{v_{0}, \ldots, v_{k}\right\}$ of $k+1$ distinct isotopy classes of essential simple closed curves to be a $k$-simplex if the isotopy classes can be represented by pairwise disjoint curves. We stress that $\mathcal{C}^{\Delta}(S)$ is an abstract simplicial complex and so its elements are simplices. Inclusion of faces induces a natural partial order on $\mathcal{C}^{\Delta}(S)$. The simplicial curve complex of $(S, z)$ is similarly denoted $\mathcal{C}^{\Delta}(S, z)$

Following the nomenclature of the subject, we let $\mathcal{C}(S)$ and $\mathcal{C}(S, z)$ denote the curve complexes of $S$ and $(S, z)$, respectively. These are geodesic metric spaces obtained by isometrically gluing regular Euclidean simplices with all edge lengths equal to one according to the combinatorics of the associated abstract simplicial complex (compare [6, I.7]). The necessity for distinguishing between $\mathcal{C}$ and $\mathcal{C}^{\Delta}$ will soon become apparent.

In the case that $\xi(S)=1, \mathcal{C}^{\Delta}(S)$ is zero dimensional and one often makes a separate definition for the curve complexes in these cases. However, we do not do this here, and so considering $\mathcal{C}(S)$ a geodesic metric space is nonsensical - a geodesic metric would have to assign an "infinite distance" to any two points. In this special case, we simply treat $\mathcal{C}(S)$ as a countable set of points. As we will only consider metric properties of $\mathcal{C}(S, z)$, and not $\mathcal{C}(S)$, this is not a serious issue. For this reason, and so we need not continue to comment on the special case, we discuss the relevant metric geometry of $\mathcal{C}(S, z)$ only.

The 1-skeleton $\mathcal{C}^{1}(S, z)$ is itself a metric space (with the induced geodesic metric), and the inclusion into $\mathcal{C}(S, z)$ is a quasiisometry. Because a geodesic in $\mathcal{C}^{1}(S, z)$ between vertices has a combinatorial description as a sequence of adjacent vertices, we may mix combinatorial and geometric arguments in the metric space $\mathcal{C}^{1}(S, z)$. We will therefore work with the metric on $\mathcal{C}^{0}(S, z)$ induced by the inclusion into $\mathcal{C}^{1}(S, z)$, which takes integer values only. 
A simplex $v \in \mathcal{C}^{\Delta}$ determines a subset $v \subset \mathcal{C}$-its realization in $\mathcal{C}$-as well as a union of curves on the surface $v \subset S$, realizing the isotopy class determined by $v$. To avoid burdening the reader with additional notation, will write $v$ for all of these with the context determining the particular meaning.

For clarity, we will typically denote simplices of $\mathcal{C}^{\Delta}(S, z)$ by $u=\left\{u_{0}, \ldots, u_{k}\right\}$ and simplices of $\mathcal{C}^{\Delta}(S)$ by $v=\left\{v_{0}, \ldots, v_{k}\right\}$. If $v$ is a simplex in $\mathcal{C}^{\Delta}(S)$, we will write $[v]$ for the geodesic representative of $v$, which is a union of pairwise disjoint embedded simple closed geodesics in $S$.

\subsection{Forgetful projection}

Any simple closed curve $u$ in $(S, z)$ can be viewed as a curve in $S$ which we denote $\Pi(u)$. However, if $S$ has punctures then an essential curve in $(S, z)$ may become inessential in $S$ by becoming peripheral. The only time this can happen is when the curve is the boundary of a once-punctured disk $(Y, z) \subset(S, z)$ containing the marked point. We call such a curve $u$ in $(S, z)$ preperipheral. A simplex $u=\left\{u_{0}, \ldots, u_{k}\right\} \in \mathcal{C}^{\Delta}(S, z)$ is called preperipheral if one of its vertices $u_{i}$ is a preperipheral curve in $(S, z)$, and nonpreperipheral otherwise.

We define the nonpreperipheral subcomplex of $\mathcal{C}^{\Delta}(S, z)$ by

$$
\widehat{C}^{\Delta}(S, z)=\left\{u \in \mathcal{C}^{\Delta}(S, z) \mid u \text { is nonpreperipheral }\right\} .
$$

There is now a well-defined simplicial map

$$
\Pi_{\Delta}: \widehat{\mathcal{C}}^{\Delta}(S, z) \rightarrow \mathcal{C}^{\Delta}(S)
$$

determined by forgetting the marked point $z$. We also write

$$
\Pi: \widehat{\mathcal{C}}(S, z) \rightarrow \mathcal{C}(S)
$$

for the induced map on metric spaces (or sets if $\xi(S)=1$ ).

Lemma 2.1. If $u \subset \widehat{\mathcal{C}}(S, z)$ is a $k$-simplex, then $\Pi(u)$ has dimension at least $k-1$.

If $\left.\Pi\right|_{u}$ is noninjective (so that $\Pi(u)$ has dimension $k-1$ ) and after reordering the vertices of $u$ we have $\Pi\left(u_{0}\right)=\Pi\left(u_{1}\right)$, then the curves $u_{0}$ and $u_{1}$ cobound an annulus $(Y, z) \subset(S, z)$ containing $z$.

Proof. Every simplex $u \in \widehat{C}^{\Delta}(S, z)$ is contained in a maximal simplex $u^{\prime}$ in $C^{\Delta}(S, z)$, which determines a pants decomposition (of $S \backslash\{z\}$ ). So $u^{\prime}$ has dimension $\xi(S \backslash\{z\})-1=\xi(S)$. Since $\xi(S) \geq 1$, an easy argument allows us to assume that $u^{\prime} \in \widehat{\mathcal{C}}(S, z)$.

Every component of $(S \backslash\{z\}) \backslash u^{\prime}$ is a pair of pants, exactly one of which contains the $z$-puncture. So the corresponding component $(Y, z) \subset(S, z)$ of $S \backslash u^{\prime}$ is an annulus with two boundary components. After reordering the vertices we may assume these are $u_{0}, u_{1} \in u^{\prime}$. Clearly $\Pi\left(u^{\prime}\right)$ is a pants decomposition of $S$, and so has dimension $\xi(S)-1$, one less than that of $u$. Since $\left.\Pi\right|_{\left\{u_{0}, u_{1}\right\}}$ is not 
injective, it follows that $\left.\Pi\right|_{u}$ will be noninjective if and only if it contains both $u_{0}$ and $u_{1}$, which thus cobound the annulus $(Y, z)$. In any case, the dimension can be at most one less than that of $u$.

We say that a simplex $u$ of $\widehat{\mathcal{C}}(S, z)$ is injective if $\left.\Pi\right|_{u}$ is injective and noninjective otherwise.

\section{Subsurfaces}

Consider a compact $\pi_{1}$-injective subsurface $(Y, z) \subset(S, z)$ with $Y \neq D^{2}$ and $\pi_{1}(Y, z)<\pi_{1}(S, z)$ a proper subgroup. The boundary $\partial Y$ is a disjoint union of nontrivial simple closed curves in $(S, z)$. It can happen that some of the components in $\partial Y$ are isotopic to each other in $(S, z)$ and that some of the components are peripheral in $(S, z)$. We let $\partial_{0} Y$ denote the $\operatorname{simplex}$ in $\mathcal{C}^{\Delta}(S, z)$ obtained by identifying pairs of components of $\partial Y$ that are isotopic in $(S, z)$ and forgetting the peripheral components.
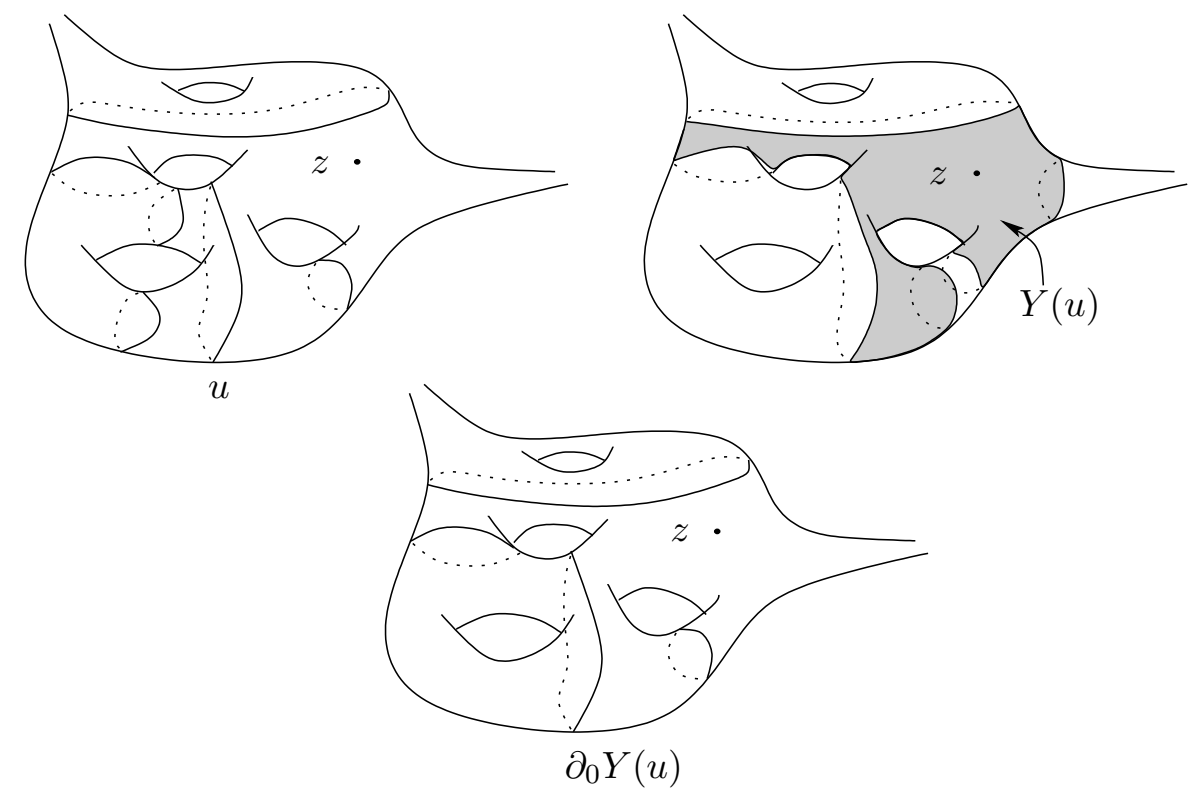

Figure 1: A simplex $u \in \mathcal{C}^{\Delta}(S, z)$, its subsurface $(Y(u), z)$, and $\partial_{0} Y(u) \subseteq u$.

Let $u \in \mathcal{C}^{\Delta}(S, z)$ be any simplex. We construct a subsurface $(Y(u), z) \subset$ $(S, z)$ as follows. Remove from $S$ a small open tubular neighborhood of $u$ and small open cusp neighborhoods of the punctures (in particular, these neighborhoods should be pairwise disjoint and not contain $z$ ), and let $Y(u)$ be the component of this subsurface containing $z$. Note that $\partial_{0} Y(u) \subseteq u$ is a potentially proper face - see Figure 1 for an example. We can iterate this process of 
taking subsurface, then $\partial_{0}$, but it immediately stabilizes: $Y\left(\partial_{0} Y(u)\right)$ is isotopic fixing $z$ to $Y(u)$.

Lemma 3.1. If $u \in \mathcal{C}(S, z)$, then $(Y(u), z)$ is an annulus (containing $z$ ) if and only if $u$ is either preperipheral or nonpreperipheral and noninjective. Furthermore, in this case $u$ is preperipheral if and only if the boundary components of $Y(u)$ are peripheral in $S$.

Proof. If $u \in \widehat{\mathcal{C}}^{\Delta}(S, z)$, the lemma follows immediately from Lemma 2.1 and the definitions.

All that remains is to prove that for a preperipheral simplex $u \in \mathcal{C}^{\Delta}(S, z)$, $Y(u)$ is an annulus for which the boundary components are peripheral in $S$. Let $u_{0} \in u$ be the preperipheral vertex. It follows that $u$ bounds a once-punctured disk containing $z$, that is, $Y\left(u_{0}\right)$ is an annulus of the required type. Now let $u_{1} \in u$ be any other vertex. The curve $u_{1}$ cannot be contained in $(Y(u), z)$, since $Y(u)-\{z\}$ is a pair of pants and thus contains no essential simple closed curves. Therefore, all other components of $u$ lie outside $Y\left(u_{0}\right)$, and so $Y\left(u_{0}\right)=Y(u)$, completing the proof.

\subsection{From simplices to groups}

To each subsurface $(Y, z) \subset(S, z)$ described in the previous section we can associate its fundamental group $\pi_{1}(Y, z)<\pi_{1}(S, z)$. We let $\mathcal{D}$ denote the collection of all such subgroups and define a map

$$
\Gamma: \mathcal{C}^{\Delta}(S, z) \rightarrow \mathcal{D}
$$

by declaring that $\Gamma(u)=\pi_{1}(Y(u), z)<\pi_{1}(S, z)$. Note that while $(Y(u), z)$ is only defined up to isotopy fixing $z$ (since $u$ is), $\Gamma(u)$ is a well defined subgroup of $\pi_{1}(S, z)$.

The set $\mathcal{D}$ admits a natural partial order by inclusion as well as an action of $\pi_{1}(S, z)$ by conjugation.

Proposition 3.2. The map $\Gamma$ is a $\pi_{1}(S, z)$-equivariant order-reversing surjection.

Proof. Given a subgroup $\pi_{1}(Y, z)<\pi_{1}(S, z)$ in $\mathcal{D}$, we have $\pi_{1}\left(Y\left(\partial_{0} Y\right), z\right)=$ $\pi_{1}(Y, z)$, and hence $\Gamma\left(\partial_{0} Y\right)=\pi_{1}(Y, z)$. So $\Gamma$ is surjective. If $u \subseteq u^{\prime}$ is a face, then $Y(u) \supseteq Y\left(u^{\prime}\right)$, and so $\pi_{1}(Y(u), z) \geq \pi_{1}\left(Y\left(u^{\prime}\right), z\right)$. In other words, $\Gamma(u) \geq \Gamma\left(u^{\prime}\right)$. So $\Gamma$ is order-reversing.

If $\gamma \in \pi_{1}(S, z)$, then $Y\left(h_{\gamma} u\right)=h_{\gamma}(Y(u))$. We have

$$
\begin{aligned}
\Gamma\left(h_{\gamma} u\right)=\pi_{1}\left(Y\left(h_{\gamma} u\right), z\right) & =\pi_{1}\left(h_{\gamma}(Y(u)), z\right) \\
& =\gamma \pi_{1}(Y(u), z) \gamma^{-1} \quad \text { by }(1) \\
& =\gamma \Gamma(u) \gamma^{-1}
\end{aligned}
$$

and it follows that $\Gamma$ is equivariant. 


\subsection{From groups to convex hulls}

In the following, all stabilizers are taken with respect to actions of $\pi_{1}(S)$ and are denoted $\operatorname{Stab}(\cdot)$. Our choice of isomorphism $\pi_{1}(S) \cong \pi_{1}(S, z)$ allows us to view $\mathcal{D}$ as a collection of subgroups of $\pi_{1}(S)$ acting on the hyperbolic plane $\widetilde{S}$. The type of the group $\Gamma(u)$ is reflected by the type of the simplex $u$. More precisely, from the definitions and Lemma 3.1 we have the following:

(i) $\Gamma(u)$ is a nonabelian free group if and only if $u$ is nonpreperipheral and $u$ is injective.

(ii) $\Gamma(u)$ is cyclic generated by a hyperbolic element if and only if $u$ is nonpreperipheral and $u$ is noninjective.

(iii) $\Gamma(u)$ is cyclic generated by a parabolic element if and only if $u$ is preperipheral.

In cases (i) and (ii), we let $\operatorname{Hull}(\Gamma(u))$ denote the convex hull of the limit set $\Lambda_{\Gamma(u)}$ of $\Gamma(u)$ acting on $\widetilde{S} \cong \mathbb{H}^{2}$. In case (ii), $\Gamma(u)$ is cyclic and $\operatorname{Hull}(\Gamma(u))$ is the axis of the elements of $\Gamma(u)$. Alternatively, $\operatorname{Hull}(\Gamma(u))$ is a component of $p^{-1}([\Pi(u)])$ (recall that $[\Pi(u)]$ is the geodesic representative of $\Pi(u)$ ). Note that $\Gamma(u)=\operatorname{Stab}(\operatorname{Hull}(\Gamma(u)))$ since the natural map of the quotient $\operatorname{Hull}(\Gamma(u)) / \Gamma(u)=[\Pi(u)]$ into $S$ is injective.

If $\Gamma(u)$ is not cyclic (case (i)), then $\operatorname{Hull}(\Gamma(u))$ is a subsurface of $\widetilde{S}$ bounded by geodesics. Consider the isotopy (not fixing $z$ ) from $\Pi(u)$ to the geodesic representative $[\Pi(u)]$ in $S$. This takes $Y(u)$ to a compact core for the quotient of the interior $\operatorname{int}(\operatorname{Hull}(\Gamma(u))) / \Gamma(u) \subset S$. This can be seen by lifting the isotopy to a $\pi_{1}(S)$-equivariant isotopy in $\widetilde{S}$ taking $p^{-1}(\Pi(u))$ to $p^{-1}([\Pi(u)])$. Because int $(\operatorname{Hull}(\Gamma(u))) / \Gamma(u)$ injects into $S$ and since $\operatorname{Stab}(\operatorname{Hull}(\Gamma(u)))=$ $\operatorname{Stab}(\operatorname{int}(\operatorname{Hull}(\Gamma(u))))$ it follows that $\Gamma(u)=\operatorname{Stab}(\operatorname{Hull}(\Gamma(u)))$.

In case (iii), the convex hull of the limit set of $\Gamma(u)$ is empty since the limit set is a single point. Here we define $\operatorname{Hull}(\Gamma(u))$ to be a $\Gamma(u)$-invariant horoball, chosen as follows. We choose a $\pi_{1}(S)$-invariant family of horoballs, one centered at each parabolic fixed point, with the property that for any horoball in the family

(I) the quotient by the stabilizer embeds as a cusp neighborhood of the associated puncture in $S$

and

(II) the boundary of the cusp neighborhood (which is the quotient of the boundary horocycle by the stabilizer) is disjoint from every simple closed geodesic in $S$.

That this is possible is a well-known consequence of Jørgensen's inequality (more precisely, the Shimizu-Leutbecher Lemma [16, II.C]). In Section 6 we impose tighter restrictions on these horoballs, but, for now, this suffices.

Note that we also have $\operatorname{Stab}(\operatorname{Hull}(\Gamma(u)))=\Gamma(u)$ in case (iii) since $\Gamma(u)$ is necessarily a maximal parabolic subgroup. We therefore have 
Lemma 3.3. For every $\Gamma(u) \in \mathcal{D}$, $\operatorname{Stab}(\operatorname{Hull}(\Gamma(u)))=\Gamma(u)$.

Let $N(\Gamma(u))$ denote the normalizer of $\Gamma(u)$ in $\pi_{1}(S)$. Lemma 3.3 yields

Proposition 3.4. For every $\Gamma(u) \in \mathcal{D}, N(\Gamma(u))=\Gamma(u)$.

Proof. As is true for any subgroup of $\pi_{1}(S)$, we have

$$
\Gamma(u) \leq N(\Gamma(u)) \leq \operatorname{Stab}\left(\Lambda_{\Gamma(u)}\right)
$$

and

$$
\operatorname{Stab}\left(\Lambda_{\Gamma(u)}\right)=\operatorname{Stab}(\operatorname{Hull}(\Gamma(u))) .
$$

Combining these relations with Lemma 3.3 we have

$$
\Gamma(u) \leq N(\Gamma(u)) \leq \Gamma(u),
$$

and the proposition follows.

We have associated to each $\Gamma(u) \in \mathcal{D}$ a convex set $\operatorname{Hull}(\Gamma(u))$ and we let $\mathcal{H}$ denote the set of all such convex sets. We order $\mathcal{H}$ by inclusion and note that $\pi_{1}(S)$ clearly acts on $\mathcal{H}$ as convex hulls of limit sets are natural and our set of horoballs is $\pi_{1}(S)$-invariant.

Lemma 3.5. The map Hull: $\mathcal{D} \rightarrow \mathcal{H}$ is a $\pi_{1}(S)$-equivariant order-preserving surjection.

Proof. First observe that $\Gamma(u) \leq \Gamma\left(u^{\prime}\right)$ implies $\Lambda_{\Gamma(u)} \subseteq \Lambda_{\Gamma\left(u^{\prime}\right)}$.

If neither group $\Gamma(u)$ nor $\Gamma\left(u^{\prime}\right)$ is parabolic, then it immediately follows that $\operatorname{Hull}(\Gamma(u)) \subseteq \operatorname{Hull}\left(\Gamma\left(u^{\prime}\right)\right)$. If both groups are parabolic, then we must have $\Gamma(u)=\Gamma\left(u^{\prime}\right)$, for the parabolic subgroups of $\mathcal{D}$ are maximal parabolic subgroups, and so $\operatorname{Hull}(\Gamma(u))=\operatorname{Hull}\left(\Gamma\left(u^{\prime}\right)\right)$.

The only remaining case is when $\Gamma(u)$ is parabolic and $\Gamma\left(u^{\prime}\right)$ is not. Since $\Gamma(u)<\Gamma\left(u^{\prime}\right)$, some $\Gamma(u)$-invariant horoball is contained in $\operatorname{Hull}\left(\Gamma\left(u^{\prime}\right)\right)$. Since Hull $\left(\Gamma\left(u^{\prime}\right)\right)$ is bounded by geodesics that descend to simple closed geodesics in $S$, and these geodesics are disjoint from the boundaries of the cusps $\operatorname{Hull}(\Gamma(u)) / \Gamma(u)$ by construction, it follows that $\operatorname{Hull}(\Gamma(u)) \subseteq \operatorname{Hull}\left(\Gamma\left(u^{\prime}\right)\right)$.

The map is surjective by construction.

Proposition 3.2 and Lemma 3.5 have the following corollary.

\section{Corollary 3.6.}

$$
\text { Hull } \circ \Gamma: \mathcal{C}^{\Delta}(S, z) \rightarrow \mathcal{H}
$$

is a $\pi_{1}(S)$-equivariant order-reversing surjection. 


\section{Stabilizers and Kra's Theorem}

Theorem 4.1. If $u \in \mathcal{C}^{\Delta}(S, z)$ is any simplex, then

$$
\operatorname{Stab}(u)=\Gamma(u)
$$

with respect to the action determined by the inclusion $\pi_{1}(S, z)<\operatorname{Mod}(S, z)$.

We note that the stabilizer in $\pi_{1}(S, z)<\operatorname{Mod}(S, z)$ of a simplex $u$ fixes that simplex pointwise since $\pi_{1}(S, z)$ acts trivially on homology, and so by Theorem 1.2 of [12], it consists entirely of pure mapping classes.

Proof. One inclusion is obvious. Namely,

$$
\Gamma(u)=\pi_{1}(Y(u), z) \leq \operatorname{Stab}(u)
$$

for if $\gamma \in \pi_{1}(Y(u), z)$, then the diffeomorphism $h_{\gamma}$ can be chosen to be supported on $Y(u)$. In particular, $h_{\gamma}$ fixes $u$.

To prove the other inclusion, observe that the $\pi_{1}(S, z)$-equivariance of $\Gamma$ implies that the stabilizer of $u$ is contained in the stabilizer of $\Gamma(u)$. Since $\pi_{1}(S, z)$ acts by conjugation on $\mathcal{D}$ this means that

$$
\operatorname{Stab}(u) \leq N(\Gamma(u)) .
$$

Proposition 3.4 implies $N(\Gamma(u))=\Gamma(u)$ and so

$$
\operatorname{Stab}(u) \leq \Gamma(u)
$$

as required.

We obtain Kra's Theorem as a corollary:

Theorem 4.2 (Kra). Given $\gamma \in \pi_{1}(S, z), h_{\gamma} \in \operatorname{Mod}(S, z)$ is pseudo-Anosov if and only if $\gamma$ is filling.

An element of $\pi_{1}(S, z)$ is filling if every representative loop nontrivially intersects every essential closed curve in $S$. We note that every representative loop of an element $\gamma \in \pi_{1}(S, z)$ intersects every essential closed curve in $S$ if every closed curve in the free homotopy class of $\gamma$ does.

Proof. Since $h_{\gamma}$ is pure, it is pseudo-Anosov if and only if it does not stabilize any simplex. By Theorem 4.1 this happens if and only if $\gamma$ is not in any group $\pi_{1}(Y(u), z)$ for any $u \in \mathcal{C}^{\Delta}(S, z)$.

We claim that $\gamma$ is not in any group $\pi_{1}(Y(u), z)$ for any $u \in C^{\Delta}(S, z)$ if and only if $\gamma$ is filling. To see this, first observe that $\gamma$ is in $\pi_{1}(Y(u), z)$ if and only if it can be realized disjoint from $\partial_{0} Y(u)$. If $\partial_{0} Y(u)$ is not preperipheral, then $\gamma$ has a representative disjoint from any component of $\Pi\left(\partial_{0} Y(u)\right)$, which is an essential curve in $S$. If $\partial_{0} Y(u)$ is preperipheral, then $\gamma$ is a peripheral loop, so has a representative disjoint from a representative of any essential closed curve in $S$. 


\section{Purely pseudo-Anosov subgroups}

Let $G<\pi_{1}(S, z)$ be a finitely generated subgroup, purely pseudo-Anosov when considered a subgroup of $\operatorname{Mod}(S, z)$. We view $G$ as a subgroup of $\pi_{1}(S)$ acting as a fuchsian group on the hyperbolic plane $\widetilde{S}$ and make uniform estimates to be used in the proof of Theorem 6.1.

By Theorem 4.2, the free homotopy class in $S$ defined by any nontrivial element of $G$ fills $S$. Let $\Sigma=\operatorname{Hull}(G) / G$ be the quotient hyperbolic surface with geodesic boundary and let

$$
p_{0}: \operatorname{Hull}(G) \rightarrow \Sigma
$$

be the covering projection.

The surface $\Sigma$ is compact. To see this, note that finite generation of fuchsian groups is equivalent to geometric finiteness (see Theorem 10.1.2 of [1]) and that $G$ is purely hyperbolic as a fuchsian group acting on $\widetilde{S}$, as every element corresponds to a filling loop on $S$.

The inclusion $\operatorname{Hull}(G) \rightarrow \widetilde{S}$ induces an immersion $f: \Sigma \rightarrow S$ with $f_{*}\left(\pi_{1}(\Sigma)\right)=$ $G$. We collect our maps into a commuting diagram:

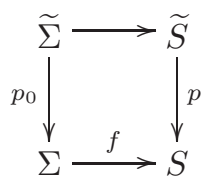

Since every nontrivial conjugacy class in $G$ is filling, for any geodesic $[v]$ in $S, f^{-1}([v])$ cuts $\Sigma$ into disks. To see this, note that if this were not the case, then there would be a nontrivial loop $\gamma \in \pi_{1}(\Sigma)$ disjoint from $f^{-1}([v])$, and so $f_{*}(\gamma)$ could not be filling as it would be disjoint from $v$. Moreover, as we will see, the family of arcs of $f^{-1}([v])$ as $v$ ranges over all of $\mathcal{C}^{0}(S)$ is a precompact family.

It will be convenient in the proof of the lower bound for Theorem 6.3 to demonstrate precompactness of the family in a slightly larger surface. Namely, let $\Sigma_{1}=N_{1}(\operatorname{Hull}(G)) / G$ denote the quotient of the 1-neighborhood of $\operatorname{Hull}(G)$ by $G$. This adds a collar of width one to each boundary component of $\Sigma$. There is an obvious extension of $f$ to $\Sigma_{1} \supset \Sigma$ that we still denote $f: \Sigma_{1} \rightarrow S$. Let $\mathcal{A}$ denote the set of all arcs of $f^{-1}([v])$ in $\Sigma_{1}$ as $v$ ranges over all of $\mathcal{C}^{0}(S)$.

Proposition 5.1. There are only finitely many isotopy classes in $\mathcal{A}$ and there is a uniform upper bound on the length of any arc in $\mathcal{A}$.

Proof. The first statement clearly follows from the second.

Suppose that there is a sequence $\left\{v_{n}\right\} \subset \mathcal{C}^{0}(S)$ and components $L_{n} \subset$ $f^{-1}\left(\left[v_{n}\right]\right)$ so that $\ell\left(L_{n}\right) \rightarrow \infty$. After passing to a subsequence, we may assume that $\left[v_{n}\right]$ has a Hausdorff limit $\lambda$, a geodesic lamination on $S$. Let $\lambda^{\prime}$ be the maximal measurable sublamination of $\lambda$, obtained from $\lambda$ by throwing away all non-closed isolated leaves. 
After passing to a further subsequence if necessary, we assume that $L_{n}$ has a Hausdorff limit, necessarily contained in $f^{-1}(\lambda)$. Since $\ell\left(L_{n}\right) \rightarrow \infty$, there is a connected geodesic lamination $\kappa$ contained in this limit and $f(\kappa)$ is a component of $\lambda^{\prime}$-the components of $\lambda^{\prime}$ are exactly the sublaminations. If $\kappa$ is a simple closed geodesic, then $f(\kappa) \subset \lambda^{\prime}$ represents a conjugacy class in $G$ which is not filling and we obtain a contradiction. So $\kappa$ is not a simple closed geodesic.

Let $W_{\kappa}$ denote the supporting subsurface of $\kappa$-the smallest open, locally convex subsurface containing $\kappa$-and let $Y_{f(\kappa)}$ be the supporting subsurface of $f(\kappa)$. Since the supporting subsurface is determined by the preimage of the lamination in the universal cover, it easily follows that

$$
f\left(W_{\kappa}\right)=Y_{f(\kappa)}
$$

Thus the surface $f\left(W_{\kappa}\right)$ is the component of the supporting subsurface of $\lambda^{\prime}$ containing $f(\kappa)$ and $f\left(\partial W_{\kappa}\right)$ is disjoint from $\lambda^{\prime}$. This is impossible since every curve representing a conjugacy class of $G$ intersects every lamination in $S$, and any component of $f\left(\partial W_{\kappa}\right)$ represents a conjugacy class in $G$.

Note that each component of $\Sigma_{1} \backslash f^{-1}([v])$ is a not only a disk, but a disk with uniformly bounded diameter (with respect to the induced path metric): it is convex, and has uniformly bounded circumference. This implies the same statement for the disks $\overline{N_{1}(\operatorname{Hull}(G)) \backslash p^{-1}([v])}$, which are just the disks of $\overline{p_{0}^{-1}\left(\Sigma_{1} \backslash f^{-1}([v])\right)}$. Moreover, since $N_{1}(\operatorname{Hull}(G)) \cap p^{-1}([v])=p_{0}^{-1}\left(\Sigma_{1} \cap f^{-1}([v])\right)$, the lemma tells us that these arcs have uniformly bounded diameter also.

The components of $p^{-1}([v])$ and the closures of the components of $\widetilde{S} \backslash p^{-1}([v])$ are precisely the convex hulls $\operatorname{Hull}(\Gamma(u))$ for $\Gamma(u) \in \mathcal{D}$ with $\Pi_{\Delta}(u)=v$. With the remarks of the previous paragraph and Proposition 5.1, this implies the following.

Corollary 5.2. There exists a $D>0$ so that for any simplex $u \in \widehat{\mathcal{C}}^{\Delta}(S, z)$,

$$
\operatorname{diam}\left(\operatorname{Hull}(\Gamma(u)) \cap N_{1}(\operatorname{Hull}(G))\right) \leq D
$$

\section{Convex cocompactness}

Theorem 6.1. If $\xi(S) \geq 1$ and $G<\pi_{1}(S, z)$ is finitely generated and purely pseudo-Anosov as a subgroup of $\operatorname{Mod}(S, z)$, then $G$ is convex cocompact.

Fix a purely pseudo-Anosov subgroup $G<\pi_{1}(S, z)<\operatorname{Mod}(S, z)$ and let $\operatorname{Hull}(G) \subset N_{1}(\operatorname{Hull}(G)) \subset \widetilde{S}$ and $f: \Sigma_{1} \rightarrow S$ be as in the previous section. We assume that for any preperipheral simplex $u \in \mathcal{C}^{\Delta}(S, z)$ we have chosen $\operatorname{Hull}(\Gamma(u))$ so that

$$
\operatorname{Hull}(\Gamma(u)) \cap N_{1}(\operatorname{Hull}(G))=\emptyset .
$$

This is possible since $f\left(\Sigma_{1}\right)$ is a compact subset of $S$, and there are only finitely many such conjugacy classes of subgroups $\Gamma(u)$ (recall from $\S 3.2$ that the $\Gamma(u)$ 
are precisely the maximal parabolic subgroups and the $\operatorname{Hull}(\Gamma(u))$ are invariant horoballs).

From this choice and Corollary 5.2, we obtain the following refinement of that corollary.

Proposition 6.2. There exists a $D>0$ so that for any simplex $u \in \mathcal{C}^{\Delta}(S, z)$,

$$
\operatorname{diam}\left(\operatorname{Hull}(\Gamma(u)) \cap N_{1}(\operatorname{Hull}(G))\right) \leq D .
$$

Proof. Let $D$ be as in Corollary 5.2. If $u \in \widehat{\mathcal{C}}^{\Delta}(S, z)$, then the bound on diameter is precisely the conclusion of Corollary 5.2. If $u \in \mathcal{C}^{\Delta}(S, z)-\widehat{\mathcal{C}}^{\Delta}(S, z)$, then $u$ is preperipheral and so by our choice of horoball $\operatorname{Hull}(\Gamma(u)) \cap N_{1}(\operatorname{Hull}(G))=\emptyset$.

Fix a vertex $u \in \mathcal{C}^{0}(S, z)$ and a point $x \in \operatorname{Hull}(\Gamma(u)) \cap \operatorname{Hull}(G)$. A finite generating set for $G$ defines a word metric on $G$, but it is more convenient to use the metric

$$
\mathrm{d}_{G}(g, h):=\mathrm{d}_{\operatorname{Hull}_{(G)}}(g(x), h(x))=\mathrm{d}_{\widetilde{S}}(g(x), h(x))
$$

which is quasiisometric to any such word metric by the Milnor-Švarc Lemma. The following implies Theorem 6.1.

Theorem 6.3. The orbit map $G \rightarrow G \cdot u$ given by $g \mapsto g \cdot u$ is a quasiisometric embedding into $\mathcal{C}(S, z)$.

Proof of Theorem 6.1 from Theorem 6.3. It was shown in [13] (Theorem 1.3) and independently in [9] (Theorem 2.9) that a finitely generated subgroup of the mapping class group is convex cocompact if and only if the orbit map to the curve complex is a quasiisometric embedding.

Proof of Theorem 6.3. Write $\mathrm{d}_{1}$ for the metric on $\mathcal{C}^{0}(S, z)$ induced from the inclusion into $\mathcal{C}^{1}(S, z)$, see $\S 2.2$. We must find $K \geq 1$ and $C \geq 0$ so that for any $g \in G$, we have

$$
\frac{\mathrm{d}_{G}(\mathbf{1}, g)}{K}-C \leq \mathrm{d}_{1}(u, g \cdot u) \leq K \mathrm{~d}_{G}(\mathbf{1}, g)+C
$$

The upper bound follows from the fact that $\mathrm{d}_{G}$ is quasiisometric to the word metric, for which such an upper bound is an immediate consequence of the triangle inequality. We assume that the constants $K$ and $C$ we choose for the lower bound also suffice for the upper bound.

We proceed to the proof of the lower bound.

Let $\tau: \widetilde{S} \rightarrow \operatorname{Hull}(G)$ denote the closest point projection. This is a contraction. Moreover, a well-known fact in hyperbolic geometry is that there exists an $R>0$ so that if $\sigma$ is any geodesic segment outside $N_{1}(\operatorname{Hull}(G))$ then $\tau(\sigma)$ has length $\ell(\tau(\sigma)) \leq R$.

Next, suppose that $u^{\prime}$ is a simplex in $\mathcal{C}(S, z)$ and $\sigma$ is a geodesic segment contained in $\operatorname{Hull}\left(\Gamma\left(u^{\prime}\right)\right)$. Since $\operatorname{Hull}\left(\Gamma\left(u^{\prime}\right)\right) \cap N_{1}(\operatorname{Hull}(G))$ is convex, it cuts 
$\sigma$ into at most three geodesic segments, at most one of which is contained in $\operatorname{Hull}\left(\Gamma\left(u^{\prime}\right)\right) \cap N_{1}(\operatorname{Hull}(G))$. It follows that

$$
\ell(\tau(\sigma)) \leq 2 R+D
$$

where $D$ is as in Proposition 6.2.

Now suppose that

$$
n=\mathrm{d}_{1}(u, g \cdot u)
$$

and connect $u$ to $g \cdot u$ by a geodesic edge path $\left[u_{0}, \ldots, u_{n}\right]$. It follows from the $\pi_{1}(S)$-equivariance of Hull $\circ \Gamma$ (see Corollary 3.6) that $g(\operatorname{Hull}(\Gamma(u)))=$ $\operatorname{Hull}(\Gamma(g \cdot u))$. We construct a piecewise geodesic path

$$
\gamma:[0,2 n+1] \rightarrow \widetilde{S}
$$

connecting $x \in \operatorname{Hull}(\Gamma(u))$ to $g(x) \in g(\operatorname{Hull}(\Gamma(u)))=\operatorname{Hull}(\Gamma(g \cdot u))$ as follows.

Consider the $2 n+1$ simplices in the 1 -skeleton of $\mathcal{C}(S, z)$

$$
\begin{array}{rlrl}
w_{0}=\left\{u_{0}\right\}, & w_{1}=\left\{u_{0}, u_{1}\right\}, \\
w_{2}=\left\{u_{1}\right\}, & w_{3}=\left\{u_{1}, u_{2}\right\}, \\
w_{4}=\left\{u_{2}\right\}, & w_{5}=\left\{u_{2}, u_{3}\right\}, \\
& \vdots & & \vdots \\
w_{2 n-2}=\left\{u_{n-1}\right\}, & w_{2 n-1}=\left\{u_{n-1}, u_{n}\right\}, \\
w_{2 n}=\left\{u_{n}\right\} & &
\end{array}
$$

These have the property that

$$
w_{2 j}, w_{2 j+2} \subset w_{2 j+1}
$$

for every $j=0, \ldots, n-1$. Therefore, by Corollary 3.6 it follows that

$$
\operatorname{Hull}\left(\Gamma\left(w_{2 j}\right)\right), \operatorname{Hull}\left(\Gamma\left(w_{2 j+2}\right)\right) \supseteq \operatorname{Hull}\left(\Gamma\left(w_{2 j+1}\right)\right)
$$

for every $j=0, \ldots, n-1$. The key consequence is that

$$
\operatorname{Hull}\left(\Gamma\left(w_{k}\right)\right) \cap \operatorname{Hull}\left(\Gamma\left(w_{k+1}\right)\right) \neq \emptyset
$$

for every $k=0, \ldots, 2 n-1$.

From this it follows that we can define a path $\gamma:[0,2 n+1] \rightarrow \widetilde{S}$ with the following properties

- $\gamma(0)=x$

- $\gamma(2 n+1)=g(x)$

- $\gamma([k, k+1]) \subset \operatorname{Hull}\left(\Gamma\left(w_{k}\right)\right)$ is a geodesic segment. 
To see this, note that we have a chain of $2 n+1$ convex sets, the first containing $x$ and the last containing $g(x)$. Consecutive sets in the chain nontrivially intersect. We therefore take $\gamma([0,1])$ to be the geodesic segment from $x$ to any point of the intersection $\operatorname{Hull}\left(\Gamma\left(w_{0}\right)\right) \cap \operatorname{Hull}\left(\Gamma\left(w_{1}\right)\right)$. Next take $\gamma([1,2])$ to be the geodesic segment connecting this point to any point of $\operatorname{Hull}\left(\Gamma\left(w_{1}\right)\right) \cap$ Hull $\left(\Gamma\left(w_{2}\right)\right)$, and so on. We continue in this way, ending with a geodesic segment $\gamma([2 n, 2 n+1])$ connecting the already determined point of $\mathrm{Hull}\left(\Gamma\left(w_{2 n-1}\right)\right) \cap$ Hull $\left(\Gamma\left(w_{2 n}\right)\right)$ to $g(x)$. Convexity guarantees the last property required.

Since the geodesic segment $\gamma([k, k+1])$ is contained in $\operatorname{Hull}\left(\Gamma\left(w_{k}\right)\right)$, we see that for every $k=0, \ldots, 2 n$

$$
\ell(\tau(\gamma([k, k+1]))) \leq 2 R+D
$$

Since $\gamma$ connects $x$ to $g(x)$, so does $\tau(\gamma)$, and its length bounds the distance from $x$ to $g(x)$. Therefore we obtain

$$
\mathrm{d}_{G}(\mathbf{1}, g)=\mathrm{d}_{\operatorname{Hull}(G)}(x, g(x)) \leq \ell(\tau(\gamma)) \leq(2 n+1)(2 R+D)
$$

Isolating $n=\mathrm{d}_{1}(u, g \cdot u)$ in this inequality, we have

$$
\mathrm{d}_{1}(u, g \cdot u)=n \geq \frac{\mathrm{d}_{G}(\mathbf{1}, g)}{2(2 R+D)}-\frac{1}{2}
$$

Taking any $K \geq 2(2 R+D)$ and $C \geq 1 / 2$ completes the proof.

\section{Trees}

Given a simplex $v \in \mathcal{C}^{\Delta}(S)$, there is an associated action of $\pi_{1}(S)$ on a tree $T_{v}$, namely, the Bass-Serre tree for the splitting of $\pi_{1}(S)$ determined by $v$. We refer the reader to [19] for a general introduction to actions on trees associated to codimension-1 submanifold.

In this section we prove

Theorem 7.1. The fiber of $\Pi$ over a point $x$ in the interior of a simplex $v \subset$ $\mathcal{C}(S)$ is $\pi_{1}(S)$-equivariantly homeomorphic to the tree $T_{v}$ determined by $v$.

The fiber naturally inherits the structure of a metric simplicial tree from the point $x$, and as we vary this point in the base, we vary the metric trees continuously in the space of $\pi_{1}(S)$-trees, see $\S 7.2 .1$. Harer defined a section of $\Pi: \widehat{\mathcal{C}}(S, z) \rightarrow \mathcal{C}(S)$, and the metric on the trees can be used to parameterize a straight line deformation retraction to the image of the section. This allows us to give an alternative proof of the following theorem.

Corollary 7.2 (Harer). If $\xi(S) \geq 1$, then with respect to the polyhedral topologies, $\mathcal{C}(S)$ is homotopy equivalent to $\widehat{\mathcal{C}}(S, z)$.

Hatcher and Vogtmann have given a simplified proof of this corollary, see [11].

We begin with a discussion of the fibers of $\Pi$. 


\section{$7.1 \quad$ Fibers}

For any $x \in \mathcal{C}(S)$, the fiber $\mathcal{F}_{x}=\Pi^{-1}(x)$ can be naturally given the structure of a simplicial complex $\mathcal{F}_{x}^{\Delta}$ so that each simplex is affinely embedded in a simplex of $\widehat{\mathcal{C}}(S, z)$. Let $v$ be the unique simplex of $\mathcal{C}^{\Delta}(S)$ containing $x$ in its interior and let $\mathcal{F}_{v}^{\Delta}=\Pi_{\Delta}^{-1}(v)$ endowed with the partial order obtained by restricting the partial order on $\widehat{\mathcal{C}}^{\Delta}(S, z)$ to $\mathcal{F}_{v}^{\Delta}$. We emphasize that $\Pi_{\Delta}^{-1}(v)$ is not a simplicial subcomplex of $\widehat{\mathcal{C}}^{\Delta}(S, z)$ (unless $v \in \mathcal{C}^{0}(S)$ ), but simply the partially ordered set of simplices sent by $\Pi_{\Delta}$ to the simplex $v$.

Note that $\mathcal{F}_{x}^{\Delta}$ is $\pi_{1}(S)$-equivariantly order isomorphic to $\mathcal{F}_{v}^{\Delta}$ : the isomorphism is given by sending a simplex of $\mathcal{F}_{x}^{\Delta}$ to the smallest simplex of $\mathcal{F}_{v}^{\Delta}$ containing it.

\subsection{The trees}

We now recall one construction of the tree $T_{v}$ by constructing its underlying simplicial complex $T_{v}^{\Delta}$. The simplicial tree $T_{v}^{\Delta}$ is the tree dual to the preimage $p^{-1}([v]) \subset \widetilde{S}$. More precisely, the vertices of $T_{v}^{\Delta}$ are in a one-to-one correspondence with components of $\widetilde{S}-p^{-1}([v])$ with two vertices joined by an edge if the closures of the corresponding components nontrivially intersect.

The edge and vertex stabilizers of $T_{v}^{\Delta}$ in $\pi_{1}(S)$ are precisely the stabilizers of the components of $p^{-1}([v])$ and of $\widetilde{S} \backslash p^{-1}([v])$, respectively. By construction, the quotients of these by their stabilizers inject as components of $[v]$ and $S \backslash[v]$. It follows from the discussion in Section 3.2 that these subgroups are all contained in $\mathcal{D}$ (falling into the two first cases (i) and (ii)). Indeed, setting $\mathcal{D}_{v}$ to be the set of all edge and vertex stabilizers of $T_{v}^{\Delta}$ we have

$$
\mathcal{D}_{v}=\{\Gamma(u) \mid \Pi(u)=v\} \subset \mathcal{D} .
$$

The group $\pi_{1}(S)$ acts on $\mathcal{D}_{v}$ by conjugation (the restriction of the action on $\mathcal{D})$. Notice that the stabilizers of two distinct vertices are distinct, and similarly for the edge stabilizers. Thus, the stabilizer determines the simplex of the tree. Therefore, since (in this setting) the stabilizer of a vertex properly contains the stabilizer of any edge having it as an endpoint, we see that the simplicial complex $T_{v}^{\Delta}$ is $\pi_{1}(S)$-equivariantly reverse-order isomorphic to $\mathcal{D}_{v}$. We record this as a proposition.

Proposition 7.3. For any simplex $v \in \mathcal{C}^{\Delta}(S)$, $T_{v}^{\Delta}$ is $\pi_{1}(S)$-equivariantly reverse-order isomorphic to $\mathcal{D}_{v}$.

The map $\Gamma$ restricts to a map

$$
\Gamma_{v}: \mathcal{F}_{v}^{\Delta} \rightarrow \mathcal{D}_{v} .
$$

given by $\Gamma_{v}=\left.\Gamma\right|_{\mathcal{F}_{v}}$.

Proposition 7.4. For any simplex $v \in \mathcal{C}^{\Delta}(S), \Gamma_{v}$ is a reverse-order bijection. 
The proof will require the following lemma.

Lemma 7.5. Suppose $(Y, z),\left(Y^{\prime}, z\right) \subset(S, z)$ are compact $\pi_{1}$-injective subsurfaces with $Y, Y^{\prime} ¥ D^{2}$ and $\pi_{1}(Y, z)=\pi_{1}\left(Y^{\prime}, z\right)<\pi_{1}(S, z)$ proper. Then there is an isotopy fixing $z$ taking $Y$ to $Y^{\prime}$.

It is easy to see that there is an isotopy taking $Y$ to $Y^{\prime}$ if we do not require such an isotopy to fix $z$. The proof of the lemma is an exercise in geometric topology, which we sketch for completeness.

Sketch. With our chosen isomorphism $\pi_{1}(S) \cong \pi_{1}(S, z)$, we view $\pi_{1}(Y, z)=$ $\pi_{1}\left(Y^{\prime}, z\right)$ as a subgroup $G<\pi_{1}(S)$ of the covering group of $p: \widetilde{S} \rightarrow S$. First, by an isotopy fixing $z$ we may assume that $Y$ and $Y^{\prime}$ meet transversely (equivalently, their boundaries meet transversely). By further isotopy fixing $z$ we may assume that $\partial Y$ meets $\partial Y^{\prime}$ in the fewest possible number of points. If these boundaries are disjoint, then we easily find annuli disjoint from $z$ which we can use to produce an isotopy taking $Y$ to $Y^{\prime}$.

Now suppose that the boundaries intersect. We describe how to find a bigon not containing $z$ bounded by arcs of $\partial Y$ and $\partial Y^{\prime}$ which can be used to reduce the number of intersection points, producing a contradiction. To this end, consider $\widetilde{Y}$ and $\widetilde{Y}^{\prime}$, the $G$-invariant components of $p^{-1}(Y)$ and $p^{-1}\left(Y^{\prime}\right)$, respectively. By assumption, $\widetilde{Y}$ and $\widetilde{Y}^{\prime}$ contain exactly the same subset of $p^{-1}(z)$, namely all $G$-translates of the chosen basepoint $\widetilde{z} \in p^{-1}(z)$ defining the isomorphism $\pi_{1}(S) \cong \pi_{1}(S, z)$. The same is true for the translate of $\widetilde{Y}$ and $\widetilde{Y}^{\prime}$ by any element of $\pi_{1}(S)$. Said differently, $p^{-1}(\partial Y)$ and $p^{-1}\left(\partial Y^{\prime}\right)$ define exactly the same partition of $p^{-1}(z)$. An innermost bigon in $\widetilde{S}$ bounded by $\operatorname{arcs}$ of $p^{-1}(\partial Y)$ and $p^{-1}\left(\partial Y^{\prime}\right)$ projects to the desired bigon in $S$.

Proof of Proposition 7.4. As we have already noted, $\Gamma_{v}$ is an order-reversing surjection. We must show that $\Gamma_{v}$ is injective.

To see this, we suppose $\Gamma(u)=\Gamma\left(u^{\prime}\right)$ with $u, u^{\prime} \in \mathcal{F}_{v}^{\Delta}$. We need to show that $u=u^{\prime}$. From the definition of $\Gamma$ we have $\pi_{1}(Y(u), z)=\pi_{1}\left(Y\left(u^{\prime}\right), z\right)$ (recall that we must realize $u$ and $u^{\prime}$ by multicurves to make sense of $Y(u)$ and $Y\left(u^{\prime}\right)$ ). By Lemma 7.5 there is an isotopy fixing $z$ taking $Y(u)$ to $Y\left(u^{\prime}\right)$. This proves that $\partial_{0} Y(u)=\partial_{0} Y\left(u^{\prime}\right)$.

Now let $u_{0} \in u$ and $u_{0}^{\prime} \in u^{\prime}$ be vertices not in $\partial_{0} Y(u)$ and $\partial_{0} Y\left(u^{\prime}\right)$, respectively, for which $\Pi\left(u_{0}\right)=\Pi\left(u_{0}^{\prime}\right)$ (if there are no such vertices, then $u=\partial_{0} Y(u)=\partial_{0} Y\left(u^{\prime}\right)=u^{\prime}$ and we are done). We must show that $u_{0}=u_{0}^{\prime}$. This is another argument in geometric topology. By an isotopy fixing $Y(u)=Y\left(u^{\prime}\right)$ first assume $u_{0}$ and $u_{0}^{\prime}$ intersect transversely in the fewest possible number of points (fewest among all curves isotopic by an isotopy fixing $Y(u)=Y\left(u^{\prime}\right)$ ). If $u_{0}$ and $u_{0}^{\prime}$ are disjoint, then because they become isotopic after forgetting $z$, they must cobound an annulus in $S$. This annulus cannot possibly contain $Y(u)=Y\left(u^{\prime}\right)$, so it may be used to produce an isotopy fixing $Y(u)=Y\left(u^{\prime}\right)$ taking $u_{0}$ to $u_{0}^{\prime}$ as required. If they are not disjoint, then there is a bigon in $S$ bounded by arcs of these curves. Of course this bigon cannot contain $Y(u)=Y\left(u^{\prime}\right)$, and so it can be used to produce an isotopy fixing $Y(u)=Y\left(u^{\prime}\right)$ 
and reducing the number of intersection points of $u_{0}$ and $u_{0}^{\prime}$, as required. Therefore $u_{0}=u_{0}^{\prime}$, so $u=u^{\prime}$, and $\Gamma_{v}$ is injective.

Proof of Theorem 7.1. Since $\Gamma_{v}$ is a reverse-order bijection and since $T_{v}^{\Delta}$ is reverse-order isomorphic to $\mathcal{D}_{v}$, it follows that we have an order-preserving bijection from $T_{v}^{\Delta}$ to $\mathcal{F}_{x}^{\Delta}$. Since these are both abstract 1-dimensional simplicial complexes, they must be isomorphic.

\subsubsection{Variation of metrics}

The simplex $v$ is naturally a space of transverse measures on its underlying 1manifold: namely, a point in $v$ is a convex combination of the unit weights on the components of this 1-manifold.

The tree $\mathcal{F}_{x}$ thus inherits a metric varying continuously in $x$ by assigning to each edge the weight of the dual curve in $x$ to which it corresponds. This metric is the same as the path metric induced by the inclusion of $\mathcal{F}_{x}$ into $\widehat{\mathcal{C}}(S, z)$.

\subsection{Harer's section and a deformation retraction}

There are many ways to construct a section of the map $\Pi: \widehat{\mathcal{C}}(S, z) \rightarrow \mathcal{C}(S)$. Harer describes one as follows. First, the union of the geodesic representatives<smiles>CCC(C)(C)C</smiles>

has measure zero. We chose our basepoint $z$ to be any point outside this union. A section is then given by $v \mapsto[v]$. This makes sense because $[v]$ lies in $S \backslash\{z\}$ and geodesics minimize intersection between pairs of curves.

Let us denote the image of this section by $\mathcal{C}^{\prime}(S)$. The map $\Pi: \widehat{\mathcal{C}}(S, z) \rightarrow \mathcal{C}(S)$ composed with this section gives a map $\Pi^{\prime}: \widehat{\mathcal{C}}(S, z) \rightarrow \mathcal{C}^{\prime}(S)$. The fibers of $\Pi^{\prime}$ are precisely the fibers of $\Pi$ and are therefore metric trees. The section provides a preferred basepoint in each, the intersection with $\mathcal{C}^{\prime}(S)$.

Any metric tree $T$ with a preferred basepoint $x$ admits a "straight line" deformation retraction to the basepoint

$$
H: T \times[0,1] \rightarrow T
$$

defined by setting $H(y, t)$ to be the unique point of the geodesic segment $[x, y]$ for which $d(x, H(y, t))=(1-t) d(x, y)$.

This determines a map

$$
H: \widehat{C}(S, z) \times[0,1] \rightarrow \widehat{\mathcal{C}}(S, z)
$$

defined on each of the pointed trees by the procedure just described.

This map is not continuous with respect to the metric topology on $\widehat{\mathcal{C}}(S, z)$. The idea is that for a vertex $u$ in $\widehat{\mathcal{C}}(S, z)$ and any $0<\epsilon<1$ one can find a point $x$ within $\epsilon$ of $u$ for which $H(\{x\} \times[0,1-\epsilon])$ is always within $\epsilon$ of $u$, while $H(u, t)$ 
is making progress toward $\mathcal{C}^{\prime}(S)$. In particular, one can construct sequences $\left\{x_{n}\right\}$ with $x_{n} \rightarrow u$ and $H\left(x_{n}, 1 / 2\right) \rightarrow u$ but $H(u, 1 / 2)$ far from $u$.

However, the polyhedral topology is more natural from the perspective of algebraic topology, and here the map $H$ is continuous.

Proposition 7.6. The map $H$ is continuous with respect to the polyhedral topologies, and hence is a deformation retraction.

Proof. With respect to the polyhedral topologies, it suffices to show that the restriction of $H$ to $u \times[0,1]$ is continuous for each $u \in \widehat{\mathcal{C}}^{\Delta}(S, z)$. The set

$$
H(u \times[0,1])
$$

is a finite subcomplex $X \subset \widehat{\mathcal{C}}(S, z)$ : it is the union of paths in the fiber of $\Pi^{\prime}$ from $u$ to $\Pi^{\prime}(u)$, and there are only finitely many combinatorial types of these paths, one for each face of $\Pi^{\prime}(u)$. Thus, the restriction becomes a map

$$
\left.H\right|_{u \times[0,1]}: u \times[0,1] \rightarrow X
$$

Since $X$ is a finite simplicial complex, it is easy to check that $\left.H\right|_{u \times[0,1]}$ is continuous. Figure 2 gives a cartoon of the general situation where the euclidean simplices have been distorted affinely.

\section{References}

[1] Alan F. Beardon. The geometry of discrete groups, volume 91 of Graduate Texts in Mathematics. Springer-Verlag, New York, 1995. Corrected reprint of the 1983 original.

[2] Mladen Bestvina. Questions in geometric group theory. http://www. math.utah. edu/ bestvina.

[3] Joan S. Birman. Mapping class groups and their relationship to braid groups. Comm. Pure Appl. Math., 22:213-238, 1969.

[4] Joan S. Birman. Braids, links, and mapping class groups. Princeton University Press, Princeton, N.J., 1974. Annals of Mathematics Studies, No. 82.

[5] Joan S. Birman and Hugh M. Hilden. On the mapping class groups of closed surfaces as covering spaces. In Advances in the theory of Riemann surfaces (Proc. Conf., Stony Brook, N.Y., 1969), pages 81-115. Ann. of Math. Studies, No. 66. Princeton Univ. Press, Princeton, N.J., 1971.

[6] Martin R. Bridson and André Haefliger. Metric spaces of non-positive curvature, volume 319 of Grundlehren der Mathematischen Wissenschaften [Fundamental Principles of Mathematical Sciences]. Springer-Verlag, Berlin, 1999. 


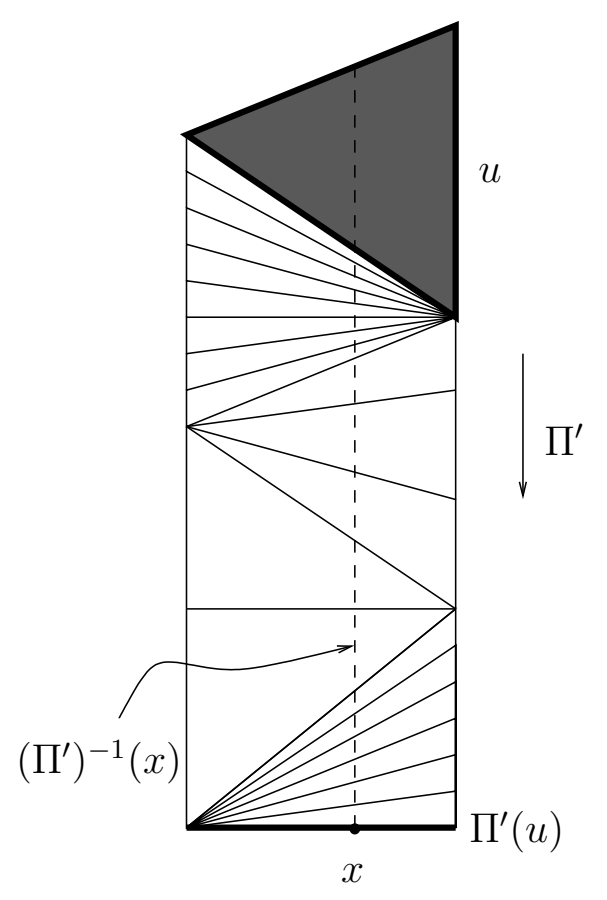

Figure 2: The deformation retraction above a simplex $\Pi^{\prime}(u)$.

[7] Max Dehn. Papers on group theory and topology. Springer-Verlag, New York, 1987. Translated from the German and with introductions and an appendix by John Stillwell, With an appendix by Otto Schreier.

[8] Benson Farb and Lee Mosher. Convex cocompact subgroups of mapping class groups. Geom. Topol., 6:91-152 (electronic), 2002.

[9] Ursula Hamenstädt. Word hyperbolic extensions of surface groups. Preprint, arXiv:math.GT/0505244.

[10] John L. Harer. The virtual cohomological dimension of the mapping class group of an orientable surface. Invent. Math., 84(1):157-176, 1986.

[11] Allen Hatcher and Karen Vogtmann. Tethers and homology stability. Preprint.

[12] Nikolai V. Ivanov. Subgroups of Teichmüller modular groups, volume 115 of Translations of Mathematical Monographs. American Mathematical Society, Providence, RI, 1992. Translated from the Russian by E. J. F. Primrose and revised by the author. 
[13] Richard P. Kent IV and Christopher J. Leininger. Shadows of mapping class groups: capturing convex cocompactness. To appear in GAFA. arXiv:math.GT/0505114.

[14] Richard P. Kent IV and Christopher J. Leininger. Subgroups of the mapping class group from the geometrical viewpoint. In the tradition of AhlforsBers, IV, 119-141. Contemp. Math. 432, American Mathematical Society, Providence, RI, 2007.

[15] Irwin Kra. On the Nielsen-Thurston-Bers type of some self-maps of Riemann surfaces. Acta Math., 146(3-4):231-270, 1981.

[16] Bernard Maskit. Kleinian groups, volume 287 of Grundlehren der Mathematischen Wissenschaften [Fundamental Principles of Mathematical Sciences]. Springer-Verlag, Berlin, 1988.

[17] Lee Mosher. Problems in the geometry of surface group extensions. In Problems on mapping class groups and related topics, volume 74 of Proc. Sympos. Pure Math., pages 245-256. Amer. Math. Soc., Providence, RI, 2006 .

[18] G. Peter Scott and Gadde A. Swarup. Geometric finiteness of certain Kleinian groups. Proc. Amer. Math. Soc., 109(3):765-768, 1990.

[19] Peter B. Shalen. Representations of 3-manifold groups. In Handbook of geometric topology, pages 955-1044. North-Holland, Amsterdam, 2002.

[20] John R. Stallings. Topologically unrealizable automorphisms of free groups. Proc. Amer. Math. Soc., 84(1):21-24, 1982.

Department of Mathematics, Brown University, Providence, RI 02912

rkent@math. brown. edu

Department of Mathematics, University of Illinois, Urbana-Champaign, IL 61801 clein@math.uiuc.edu

Department of Mathematics, University of Warwick, Coventry CV4 7AL UK s.schleimer@warwick.ac.uk 